\title{
A gradient-type deformation of conics and a class of Finslerian flows
}

\author{
Mircea CRASMAREANU
}

Dedicated to Academician Radu Miron on the occasion of his 90'th birthday

\begin{abstract}
The aim of this paper is to produce new examples of Riemannian and Finsler structures having as model a scalar deformation of conics inspired by the scaling transformation. It continues [4] from the point of view of relationship between quadratic polynomials (which provide equations of conics in dimension 2) and Finsler geometries. A type of Finslerian flow is introduced, based on the previous deformation and we completely solve the corresponding particular case of Riemannian flow.
\end{abstract}

\section{Introduction}

One of the major parts of mathematical activity of Professor R. Miron is concerning Finsler geometry. On MathSciNet there are listed 91 papers and books authored by him and dedicated to this subject. The oldest is dated 1961 and extends to arbitrary signature of the Finsler metric the techniques of Shiing-shen Chern from the paper On the Euclidean connections in a Finsler space, Proc. Nat. Acad. Sci. U. S. A., 29(1943), 33-37. The last one is [12]. The well-known book [10], co-authored with Professor M. Anastasiei, is a main reference for the Finslerian mathematicians.

Another constant attention of Academician R. Miron is dedicated to (the so-called) elementary geometry. As above, he is author of several books dedicated to both fundamentals of geometry and analytical geometry, unfortunately

Key Words: conic, invariant, Finsler fundamental function, deformation, Finslerian flow. 2010 Mathematics Subject Classification: Primary 53C60; Secondary 51MB04.

Received: June 1, 2016.

Accepted: June 27, 2016. 
most of them being in Romanian. An exception is [11] co-authored with Professor D. Brânzei.

This paper is intended to refresh the relationships between these domains of Geometry, especially from the point of view of examples. It is desirable to have several examples of Riemannian/Finsler structures or techniques to construct new Riemannian/Finsler functions from some old structures in order to obtain classes of Riemannian/Finsler geometries with special properties. So, our work develops a scalar deformation of Finsler metrics having as model a similar deformation of conics. From this point of view, we continue here the paper [4] were two-dimensional Finsler geometries are studied when their indicatrices are conics.

The paper starts with a deformation of a conic $\Gamma$ based on the components of gradient vector field for the quadratic form defining $\Gamma$. This deformation is inspired by the scaling (linear) transformation of Computer Graphics: $(x, y) \in$ $\mathbb{R}^{2} \rightarrow\left(\lambda_{x} \cdot x, \lambda_{y} \cdot y\right) \in \mathbb{R}^{2}$, following [14, p. 136]. Using the invariants from the Euclidean geometry of conics we give the classifications of the deformations.

In the next section we move to the Riemannian-Finslerian framework of arbitrary dimension and consider a deformation inspired by the previous section. We finish this paper with a type of Finslerian flows which can be the starting point of future studies following the way opened by the famous Ricci flow of Riemannian geometry, [5]. Due to the complex form of Finslerian deformation even in the Randers case, we can solve completely only the corresponding particular case of Riemannian flows. The solution is a time-dependent diagonal metric and the flow is expanding with a blow-up at the origin. Also, we point out a relationship between our setting and some recent bi-metric approaches of spacetime geometries as in [2].

Acknowledgement Special thanks are offered to Professor Vladimir Balan for several useful remarks.

\section{A gradient-type deformation of conics}

In the two-dimensional Euclidean space $\mathbb{R}^{2}$ let us consider the conic $\Gamma$ implicitly defined by $f \in C^{\infty}\left(\mathbb{R}^{2}\right)$ as: $\Gamma=\left\{(x, y) \in \mathbb{R}^{2} \mid f(x, y)=0\right\}$ where $f$ is a quadratic function of the form $f(x, y)=r_{11} x^{2}+2 r_{12} x y+r_{22} y^{2}+2 r_{10} x+$ $2 r_{20} y+r_{00}$ with $r_{11}^{2}+r_{12}^{2}+r_{22}^{2} \neq 0$.

It is well-known that the gradient vector field of $f$, namely $\nabla f=$ $\left(f_{x}=\frac{\partial f}{\partial x}, f_{y}=\frac{\partial f}{\partial y}\right)$, gives important properties of $\Gamma$; for example, the centers of $\Gamma$ are exactly the critical points of $\nabla f$. Inspired by this fact we introduce:

Definition 2.1 Fix the scalars $\alpha, \beta$ with $\alpha \beta \neq 0$. The $(\alpha, \beta)$-deformation 
of $\Gamma$ is the conic:

$$
\tilde{\Gamma}=\Gamma_{\alpha, \beta}: \alpha\left[\frac{1}{2} f_{x}\right]^{2}+\beta\left[\frac{1}{2} f_{y}\right]^{2}=0 .
$$

Examples 2.2: i) Fix other non-vanishing scalars $a, b$. The ellipse $E(a, b)$ : $\frac{x^{2}}{a^{2}}+\frac{y^{2}}{b^{2}}-1=0$ and the hyperbola $H(a, b): \frac{x^{2}}{a^{2}}-\frac{y^{2}}{b^{2}}-1=0$ have the same $(\alpha, \beta)$-deformation:

$$
E_{\alpha, \beta}=H_{\alpha, \beta}: \frac{\alpha x^{2}}{a^{4}}+\frac{\beta y^{2}}{b^{4}}=0
$$

which is the origin $O(0,0)$ for $\alpha \beta>0$ and two secant lines through $O$ if $\alpha \beta<0$. These lines are orthogonal if and only if:

$$
\frac{\alpha}{\beta}=-\frac{a^{4}}{b^{4}}
$$

and consists in the pair of canonical bisectrices: $B_{ \pm}: y= \pm x$. In particular, the $(1,-1)$-deformation of the unit circle $S^{1}$ or more generally, the $\left(a^{4},-b^{4}\right)$ deformation of $E(a, b)$, respectively $H(a, b)$, deserves this case.

Let $c \neq 0$ and $\beta=-\left(\frac{c b^{2}}{a^{2}}\right)^{2} \alpha$. Then:

$$
E_{\alpha, \beta}=H_{\alpha, \beta}: \frac{\alpha}{a^{4}}\left(x^{2}-c^{2} y^{2}\right)=0
$$

and then, $E_{\alpha, \beta}=H_{\alpha, \beta}$ is exactly the set of characteristics for the 1-dimensional homogeneous wave equation ([3, p. 54]):

$$
u_{y y}-c^{2} u_{x x}=0, \quad y>0,-\infty \leq d<x<b \leq+\infty .
$$

ii) For $p>0$ let the parabola $P(p): y^{2}-2 p x=0$. Its $(\alpha, \beta)$-deformation is:

$$
P_{\alpha, \beta}: \alpha p^{2}+\beta y^{2}=0
$$

which is the empty set for $\alpha \beta>0$ and consists in two parallel lines for $\alpha \beta<0$. iii) Consider again the ellipse $E(a, b)$ with $a>b>0$. The family of all confocal conics with $E(a, b)$ is given by:

$$
\Gamma_{\lambda}: \frac{x^{2}}{a-\lambda}+\frac{y^{2}}{b-\lambda}-1=0
$$

for $\lambda \in \mathbb{R} \backslash\{a, b\}$. The $(a-\lambda, b-\lambda)$-deformation of $\Gamma_{\lambda}$ :

$$
\left(\Gamma_{\lambda}\right)_{(a-\lambda, b-\lambda)}: \frac{x^{2}}{a-\lambda}+\frac{y^{2}}{b-\lambda}=0
$$


is exactly the homogeneous part of $\Gamma_{\lambda}$. Also, $E_{a^{2}, b^{2}}$ is the homogeneous part of $E(a, b)$.

In order to classify the $(\alpha, \beta)$-deformations we recall the algebraic invariants associated to $\Gamma$ :

$$
\begin{gathered}
\Delta=\left|\begin{array}{ccc}
r_{11} & r_{12} & r_{10} \\
r_{12} & r_{22} & r_{20} \\
r_{10} & r_{20} & r_{00}
\end{array}\right|, \\
D=\delta+r_{11} r_{00}-r_{10}^{2}+r_{22} r_{00}-r_{20}^{2}, I=r_{11}+r_{22}, \delta=r_{11} r_{22}-r_{12}^{2} .
\end{gathered}
$$

More precisely, the main result of this Section is:

Theorem 2.3 The conic $\Gamma_{\alpha, \beta}$ is a degenerated one. It is given by: 1) $\delta \neq 0, \alpha \beta>0: \Gamma_{\alpha, \beta}$ is a point,

2) $\delta \neq 0, \alpha \beta<0: \Gamma_{\alpha, \beta}$ consists in two secant lines,

3) $\delta=0, \alpha \beta>0: \Gamma_{\alpha, \beta}$ is the empty set,

4) $\delta=0, \alpha \beta<0: \Gamma_{\alpha, \beta}$ consists in two parallel lines.

Proof A straightforward computation yields the coefficients of $\tilde{\Gamma}=\Gamma_{\alpha, \beta}$ :

$$
\left\{\begin{array}{l}
\tilde{r}_{11}=\alpha r_{11}^{2}+\beta r_{12}^{2}, \tilde{r}_{12}=r_{12}\left(\alpha r_{11}+\beta r_{22}\right), \tilde{r}_{22}=\alpha r_{12}^{2}+\beta r_{22}^{2} \\
\tilde{r}_{10}=\alpha r_{11} r_{10}+\beta r_{12} r_{20}, \tilde{r}_{20}=\alpha r_{12} r_{10}+\beta r_{22} r_{20}, \tilde{r}_{00}=\alpha r_{10}^{2}+\beta r_{20}^{2}
\end{array}\right.
$$

One have:

$\tilde{I}=\alpha r_{11}^{2}+(\alpha+\beta) r_{12}^{2}+\beta r_{22}^{2}, \tilde{D}=\alpha \beta\left[\delta^{2}+\left(r_{10} r_{12}-r_{20} r_{11}\right)^{2}+\left(r_{10} r_{22}-r_{20} r_{12}\right)^{2}\right]$.

From:

$$
\left(\begin{array}{ll}
\tilde{r}_{11} & \tilde{r}_{12} \\
\tilde{r}_{12} & \tilde{r}_{22}
\end{array}\right)=\left(\begin{array}{ll}
\alpha r_{11} & \beta r_{12} \\
\alpha r_{12} & \beta r_{22}
\end{array}\right)\left(\begin{array}{ll}
r_{11} & r_{12} \\
r_{12} & r_{22}
\end{array}\right)
$$

it results:

$$
\tilde{\delta}=\alpha \beta \delta^{2}
$$

Also:

$$
\left(\tilde{r}_{i j}\right)=\left(\begin{array}{ccc}
\alpha r_{11} & \beta r_{12} & 0 \\
\alpha r_{12} & \beta r_{22} & 0 \\
\alpha r_{10} & \beta r_{20} & 0
\end{array}\right)\left(\begin{array}{ccc}
r_{11} & r_{12} & r_{10} \\
r_{12} & r_{22} & r_{20} \\
0 & 0 & 0
\end{array}\right)
$$

yields:

$$
\tilde{\Delta}=0
$$

which gives the degeneracy of $\Gamma_{\alpha, \beta}$. The hypothesis of 1) yields $\tilde{\delta}>0$ while that of 2) gives $\tilde{\delta}<0$. For 3 ) we have $\tilde{D}>0$ while 4 ) gives $\tilde{D}<0$.

Remarks 2.4 i) Returning to Examples it follows that (2.2) and (2.6) are the "canonical forms" of $(\alpha, \beta)$-deformations.

ii) The association $\Gamma \rightarrow \tilde{\Gamma}$ can be considered also from a "convex" point of 
view since $\Gamma_{\alpha, \beta}=\Gamma_{\frac{\alpha}{\sqrt{\alpha^{2}+\beta^{2}}}}, \frac{\beta}{\sqrt{\alpha^{2}+\beta^{2}}}$. Also, if instead of the usual Euclidean metric of plane we consider the (semi-) Riemannian metric $g_{\alpha, \beta}=\operatorname{diag}(\alpha, \beta)$ then the function defining $\tilde{\Gamma}$, namely $\alpha f_{x}^{2}+\beta f_{y}^{2}$, is the square norm of the gradient field $\nabla f$ with respect to $g_{\alpha, \beta}$.

iii) A special attention deserves the case $\alpha=\beta$ for which $\tilde{f}=\alpha\|\nabla f\|^{2}$. For this case we provide a relationship between some of the above invariants:

Proposition 2.5 For the conic $\Gamma$ with unique center $(i . e . \delta \neq 0)$ we define $T=\frac{I^{2}}{\delta}$. Then:

$$
\tilde{T}=(T-2)^{2} .
$$

\section{Proof From:}

$T=\frac{r_{11}^{2}+2 r_{11} r_{22}+r_{22}^{2}}{r_{11} r_{22}-r_{12}^{2}}, \quad T-2=\frac{r_{11}^{2}+r_{22}^{2}+2 r_{12}^{2}}{\delta}, \quad \tilde{T}=\frac{\left(r_{11}^{2}+r_{22}^{2}+2 r_{12}^{2}\right)^{2}}{\delta^{2}}$

we get the conclusion.

Obviously, a more general deformation is obtained with a third scalar $\gamma$ :

$$
\tilde{\Gamma}=\Gamma_{\alpha, \beta, \gamma}: \alpha\left[\frac{1}{2} f_{x}\right]^{2}+\beta\left[\frac{1}{2} f_{y}\right]^{2}+\gamma\left[\frac{1}{4} f_{x} f_{y}\right]=0 .
$$

Now we have:

$$
\left\{\begin{array}{l}
\tilde{r}_{11}=\alpha r_{11}^{2}+\beta r_{12}^{2}+\gamma r_{11} r_{12}, \tilde{r}_{12}=r_{12}\left(\alpha r_{11}+\beta r_{22}\right)+\gamma\left(r_{11} r_{22}+r_{12}^{2}\right), \\
\tilde{r}_{22}=\alpha r_{12}^{2}+\beta r_{22}^{2}+\gamma r_{12} r_{22}, \\
\tilde{r}_{10}=\alpha r_{11} r_{10}+\beta r_{12} r_{20}+\gamma r_{11} r_{20}, \tilde{r}_{20}=\alpha r_{12} r_{10}+\beta r_{22} r_{20}+\gamma r_{22} r_{10} \\
\tilde{r}_{00}=\alpha r_{10}^{2}+\beta r_{20}^{2}+\gamma r_{10} r_{20}
\end{array}\right.
$$

yielding:

$$
\left\{\begin{array}{l}
\tilde{I}=\gamma r_{12} I+\alpha r_{11}^{2}+(\alpha+\beta) r_{12}^{2}+\beta r_{22}^{2}, \\
\tilde{\delta}=\alpha \beta \delta^{2}-\left(\alpha r_{11}+\beta r_{22}\right) \gamma r_{12}\left(r_{11} r_{22}+r_{12}^{2}\right)-\gamma^{2}\left(r_{11}^{2} r_{22}^{2}+r_{11} r_{12}^{2} r_{22}+r_{12}^{4}\right) .
\end{array}\right.
$$

\section{A deformation of Finsler structures}

Let $M$ be an open subset of $\mathbb{R}^{m}$ (for a more general case see Proposition 2.3) considered as a smooth $m$-dimensional manifold with $m \geq 2$ and $\pi: T M \rightarrow M$ its tangent bundle. Let $x=\left(x^{i}\right)=\left(x^{1}, \ldots, x^{m}\right)$ be the coordinates on $M$ and $(x, y)=\left(x^{i}, y^{i}\right)=\left(x^{1}, \ldots, x^{m}, y^{1}, \ldots ., y^{m}\right)$ the induced coordinates on $T M$. Denote by $O$ the null-section of $\pi$.

Recall after [10] that a Finsler fundamental function on $M$ is a map $F$ : $T M \rightarrow \mathbb{R}_{+}$with the following properties: 
F1) $F$ is smooth on the slit tangent bundle $T_{0} M:=T M \backslash O$ and continuous on $O$,

F2) $F$ is positive homogeneous of degree 1: $F(x, \lambda y)=\lambda F(x, y)$ for every $\lambda>0$,

F3) the matrix $\left(g_{i j}\right)=\left(\frac{1}{2} \frac{\partial^{2} F^{2}}{\partial y^{i} \partial y^{j}}\right)$ is invertible and its associated quadratic form is positive definite.

The tensor field $g=\left\{g_{i j}(x, y) ; 1 \leq i, j \leq m\right\}$ is called the Finsler metric and the homogeneity of $F$ implies:

$$
F^{2}(x, y)=g_{i j} y^{i} y^{j}=y_{i} y^{i}
$$

where $y_{i}=g_{i j} y^{j}$. The pair $(M, F)$ is called Finsler manifold.

Fix now the vector $\bar{\alpha}=\left(\alpha^{1}, \ldots, \alpha^{m}\right)$ with all strictly positive components although there are cases when some of them can be null or even negative. Inspired by the previous Section we introduce:

Definition 3.1 The $\bar{\alpha}$-deformation of $F$ is $\bar{F}=F_{\bar{\alpha}}: T M \rightarrow \mathbb{R}$ given by:

$$
\bar{F}=\sqrt{\sum_{i=1}^{m} \alpha^{i}\left[\frac{1}{2}\left(F^{2}\right)_{y^{i}}\right]^{2}} .
$$

From (3.1) due to homogeneity it results a basic equation of Finsler geometry:

$$
\frac{1}{2}\left(F^{2}\right)_{y^{i}}=g_{i j} y^{j}
$$

and then the $\bar{\alpha}$-deformation of $F$ is:

$$
\bar{F}=\sqrt{\sum_{i=1}^{m} \alpha^{i}\left(g_{i j} y^{j}\right)^{2}}=\sqrt{\sum_{i=1}^{m} \alpha^{i} y_{i}^{2}} .
$$

This new Fislerian fundamental function yields a new Finslerian metric $\bar{g}=g^{\bar{\alpha}}$ which we call the $\bar{\alpha}$-deformation of $g$.

Example 3.2 (Riemannian geometry) Let $a=\left(a_{i j}(x)\right)$ be a Riemannian metric on $M$. It is well-known that $F=\sqrt{a_{i j} y^{i} y^{j}}$ is a Finslerian structure on $M$ with $g=a$. Then $\bar{F}^{2}=\sum_{i} \alpha^{i}\left(a_{i j} y^{j}\right)^{2}$ gives:

$$
\bar{F}_{y^{u}}^{2}=2 \sum_{i=1}^{m} \alpha^{i}\left(a_{i j} y^{j} a_{i u}\right)
$$

which yields the new Riemannian metric $\bar{a}$ :

$$
\bar{g}_{u v}:=\bar{a}_{u v}=\sum_{i=1}^{m} \alpha^{i} a_{i u} a_{i v} .
$$


In the particular case $m=2$ we get:

$$
\bar{a}_{11}=\alpha^{1} a_{11}^{2}+\alpha^{2} a_{12}^{2}, \bar{a}_{12}=a_{12}\left(\alpha^{1} a_{11}+\alpha^{2} a_{22}\right), \bar{a}_{22}=\alpha^{1} a_{12}^{2}+\alpha^{2} a_{22}^{2}
$$

which is exactly $\left(2.10_{1}\right)$ with $\alpha^{1}=\alpha, \alpha^{2}=\beta$ and $r \rightarrow a$.

For the general $m$, if $a$ is a diagonal Riemannian metric i.e. $a_{i j}=0$ for $i \neq j$ then $\bar{a}$ is also diagonal with:

$$
\bar{a}_{u u}=\alpha^{u} a_{u u}^{2}
$$

For example, it follows that the well-known Poincaré upper half-plane model of $m$-dimensional hyperbolic geometry ([13, p. 74]):

$$
H_{+}^{m}=\left\{x=\left(x^{1}, \ldots, x^{m}\right) \in \mathbb{R}^{m} ; x^{m}>0\right\}, \bar{a}=\frac{1}{\left(x^{m}\right)^{2}}\left(d\left(x^{1}\right)^{2}+\ldots+d\left(x^{m}\right)^{2}\right)
$$

can be considered as the $(1, \ldots, 1)$-deformation of the metric:

$$
a=\frac{1}{x^{m}}\left(d\left(x^{1}\right)^{2}+\ldots+d\left(x^{m}\right)^{2}\right)
$$

which we call $(1, \ldots, 1)$-source of the Poincaré metric. If we start with the Poincaré metric and apply the $(1, \ldots, 1)$-deformation we get another metric:

$$
\bar{a}=\frac{1}{\left(x^{m}\right)^{4}}\left(d\left(x^{1}\right)^{2}+\ldots+d\left(x^{m}\right)^{2}\right)
$$

which can be called $(1, \ldots, 1)$-target of the Poincaré metric. In follows that the Poincaré metric belongs to the discrete flow of metrics:

$$
a_{p}=\frac{1}{\left(x^{m}\right)^{p}}\left(d\left(x^{1}\right)^{2}+\ldots+d\left(x^{m}\right)^{2}\right)
$$

with $p \in\left\{2^{n}: n \in \mathbb{Z}\right\}$ and for $m=2$ the Gaussian curvature of this metric is:

$$
K_{p}\left(x^{1}, x^{2}\right)=\frac{-p}{2\left(x^{2}\right)^{2-p}}<0 .
$$

Let us remark that in [8] it is studied the metric (3.12) for $m=3$ and $p \in$ $\left\{2 n: n \in \mathbb{Z}^{*}\right\}$ from the point of view of Laplace operator.

Another important example of diagonal metric is that of $2 \mathrm{D}$ warped metric:

$$
a=d r^{2}+f^{2}(r) d \theta^{2}
$$

Then:

$$
\bar{a}=\alpha^{1} d r^{2}+\alpha^{2} f^{4}(r) d \theta^{2}
$$


and it is easy to see that $\bar{a}$ is also a warped metric: with $\bar{r}=\sqrt{\alpha^{1}} r$ and $\bar{f}(\bar{r})=\sqrt{\alpha^{2}} f^{2}\left(\frac{\bar{r}}{\sqrt{\alpha^{1}}}\right)$ we obtain that $\bar{a}=d \bar{r}^{2}+\bar{f}^{2}(\bar{r}) d \theta$.

Returning to the general $m$-dimensional case (3.8) let us remark that a diagonal metric $a$ has only three types of non-zero Christoffel symbols: $\Gamma_{u u}^{u}$, $\Gamma_{u u}^{v}, \Gamma_{u v}^{u}$. A straightforward computations yields the transformation of the Levi-Civita connections $\nabla \rightarrow \bar{\nabla}$ corresponding to the transformation (3.8):

$$
\bar{\Gamma}_{u u}^{u}=2 \Gamma_{u u}^{u}, \quad \bar{\Gamma}_{u u}^{v}=\frac{2 \alpha^{u}}{\alpha^{v}} \Gamma_{u u}^{v}, \quad \bar{\Gamma}_{u v}^{u}=2 \Gamma_{u v}^{u}
$$

which yields:

Proposition 3.3 Let $\bar{\alpha}=(1, \ldots, 1)$ and $g$ be a diagonal Riemannian metric on the general manifold $M$ in a fixed atlas. Then the possibility of $\bar{\alpha}-$ deformation yields an affine structure on $M$ i.e. the given atlas is affine.

Proof The very brief theory of affine manifolds is exposed in [1]. From (3.16) and the expression of $\bar{\alpha}$ we have that the change of Levi-Civita connections is $\bar{\nabla}=2 \nabla$. But using the well-known law of change of the Christoffel symbols at a change of charts $\left(x^{i}\right) \rightarrow\left(\tilde{x}^{a}\right)$ for both $\nabla$ and $\bar{\nabla}$ we derive that $\frac{\partial^{2} x^{i}}{\partial \tilde{x}^{a} \partial \tilde{x}^{b}}=0$ which means the conclusion.

Example 3.4 (Randers geometry) Let $F$ be a Randers fundamental function of Minkowski type:

$$
F_{b}(x, y)=F_{b}(y)=\sqrt{\left(y^{1}\right)^{2}+\ldots+\left(y^{m}\right)^{2}}+b y^{1}
$$

with $0<b<1$. The corresponding Finsler metric is:

$$
\begin{gathered}
g_{11}^{b}=1+b^{2}+b \frac{2\left(y^{1}\right)^{3}+3 y^{1} \sum_{j \geq 2}^{m}\left(y^{j}\right)^{2}}{\left(\sum_{i=1}^{m}\left(y^{i}\right)^{2}\right)^{\frac{3}{2}}}, g_{1 j}^{b}=\frac{b y^{j} \sum_{r \geq 2}\left(y^{r}\right)^{2}}{\left(\sum_{i=1}^{m}\left(y^{i}\right)^{2}\right)^{\frac{3}{2}}}, \\
g_{j j}^{b}=1+\frac{b y^{1} \sum_{i \neq j}\left(x^{i}\right)^{2}}{\left(\sum_{i=1}^{m}\left(y^{i}\right)^{2}\right)^{\frac{3}{2}}}, g_{j k}^{b}=-b \frac{y^{1} y^{j} y^{k}}{\left(\sum_{i=1}^{m}\left(y^{i}\right)^{2}\right)^{\frac{3}{2}}}
\end{gathered}
$$

for $2 \leq j, k \leq m$ and $j \neq k$. Since the computations becomes very complicated we restrict from now to $m=2$. It follows:

$$
\bar{F}^{2}=\alpha^{1}\left[\left(1+b^{2}\right) y^{1}+\frac{b\left(2\left(y^{1}\right)^{2}+\left(y^{2}\right)^{2}\right)}{\sqrt{\left(y^{1}\right)^{2}+\left(y^{2}\right)^{2}}}\right]^{2}+\frac{\alpha^{2}\left(y^{2}\right)^{2}}{\left(y^{1}\right)^{2}+\left(y^{2}\right)^{2}} F^{2} .
$$

A more detailed formula for this Finslerian fundamental function is:

$$
\bar{F}^{2}=\alpha^{1}\left[\left(1+5 b^{2}+b^{4}\right)\left(y^{1}\right)^{2}+b^{2}\left(y^{2}\right)^{2}+\frac{b^{2}\left(y^{1}\right)^{4}}{\left(y^{1}\right)^{2}+\left(y^{2}\right)^{2}}\right.
$$




$$
\begin{gathered}
\left.+2 b\left(1+b^{2}\right) y^{1} \sqrt{\left(y^{1}\right)^{2}+\left(y^{2}\right)^{2}}+\frac{\left.2 b\left(1+b^{2}\right)\left(y^{1}\right)^{3}\right)}{\sqrt{\left(y^{1}\right)^{2}+\left(y^{2}\right)^{2}}}\right]+ \\
\frac{\alpha^{2}\left(y^{2}\right)^{2}}{\left(y^{1}\right)^{2}+\left(y^{2}\right)^{2}} F^{2}
\end{gathered}
$$

which proves that this type of deformation can covers a large class of metrics. For the expression of $\bar{g}$ we give only one:

$$
\begin{aligned}
\bar{g}_{22}=\alpha^{1} b^{2}\{1 & \left.-\frac{\left(y^{1}\right)^{4}\left[\left(y^{1}\right)^{2}-\left(y^{2}\right)^{2}\right]}{\left[\left(y^{1}\right)^{2}+\left(y^{2}\right)^{2}\right]^{3}}+\frac{3\left(1+b^{2}\right)\left(y^{1}\right)^{3}\left(y^{2}\right)^{2}}{b\left[\left(y^{1}\right)^{2}+\left(y^{2}\right)^{2}\right]^{\frac{3}{2}}}\right\} \\
+ & \frac{\alpha^{2}}{2} \frac{\partial^{2}}{\partial\left(y^{2}\right)^{2}}\left[\frac{\left(y^{2}\right)^{2}}{\left(y^{1}\right)^{2}+\left(y^{2}\right)^{2}} F^{2}\right]
\end{aligned}
$$

and we remark that:

$$
\lim _{b \rightarrow 0} \bar{g}_{22}=\alpha^{1} \frac{3\left(y^{1}\right)^{3}\left(y^{2}\right)^{2}}{\left[\left(y^{1}\right)^{2}+\left(y^{2}\right)^{2}\right]^{\frac{5}{2}}}+\alpha^{2} \neq 1=g_{22}^{0} .
$$

This proves that the new Finslerian structure $\bar{F}$ defines a new Finsler geometry on $M$.

Returning to the general Finslerian case we introduce the Cartan tensor:

$$
C_{i j k}=\frac{1}{4} \frac{\partial^{3} F^{2}}{\partial y^{i} y^{j} y^{k}}
$$

The homogeneity of $F$ implies:

$$
C_{i j k} y^{k}=C_{i j k} y^{i}=C_{i j k} y^{j}=0
$$

and then:

$$
\bar{F}_{y^{u}}^{2}=2 \sum_{i=1}^{m} \alpha^{i}\left(g_{i j} y^{j}\right) g_{i u}=2 \sum_{i=1}^{m} \alpha^{i} y_{i} g_{i u} .
$$

It follows the general formula for the $\bar{\alpha}$-deformation of $g$ :

$$
\bar{g}_{u v}=\sum_{i=1}^{m} \alpha^{i}\left[g_{i u} g_{i v}+2\left(g_{i j} C_{i u v}\right) y^{j}\right]=\sum_{i=1}^{m} \alpha^{i}\left[g_{i u} g_{i v}+2 y_{i} C_{i u v}\right]
$$

and, since the Riemannian metrics are characterized by the vanishing of the Cartan tensor, we recast (3.6) in the particular case of Riemannian manifolds.

Example 3.5 (Spherically symmetric Finsler functions) Let $I \subseteq \mathbb{R}_{+}$be an interval and $A, B: I \rightarrow \mathbb{R}$ two smooth functions. Denoting by $<,>$ the 
Euclidean inner product and by $|y|$ the Euclidean norm of the vector $y$ we define the orthogonally invariant Finsler function:

$$
F(x, y)=\sqrt{A\left(|x|^{2}\right)|y|^{2}+B\left(|x|^{2}\right)<x, y>^{2}} .
$$

Its $\bar{\alpha}$-deformation is:

$\bar{F}^{2}=A^{2}\left[\sum \alpha^{i}\left(y^{i}\right)^{2}\right]+B^{2}<x, y>^{2}\left[\sum \alpha^{i}\left(x^{i}\right)^{2}\right]+2 A B<x, y>\left(\sum \alpha^{i} x^{i} y^{i}\right)$.

In particular, for $\bar{\alpha}=(1, \ldots, 1)$ we obtain:

$$
\bar{F}=\sqrt{\bar{A}|y|^{2}+\bar{B}<x, y>^{2}}, \bar{A}(t)=A^{2}(t), \bar{B}(t)=t \cdot B^{2}(t)+2 A(t) B(t) .
$$

For example, let $c \in\{+1,0,-1\}$ and the Riemannian space-form $\left(M_{c}, g^{c}\right)$ provided by the curvature equal to $c$. We have the Finsler fundamental function:

$$
F^{c}(x, y)=\frac{\sqrt{\left(1+c|x|^{2}\right)|y|^{2}-c<x, y>^{2}}}{1+c|x|^{2}}
$$

and the Riemannian metric:

$$
g_{i j}^{c}=\frac{\left(1+c|x|^{2}\right) \delta_{i j}-c x^{i} x^{j}}{\left(1+c|x|^{2}\right)^{2}} .
$$

Hence:

$$
A^{c}(t)=\frac{1}{1+c t}, \quad B^{c}(t)=\frac{-c}{(1+c t)^{2}}
$$

where for $c=-1$ the defining interval is $[0,1)$, while for $c \in\{0,+1\}$ we have $I=\mathbb{R}_{+}$. It results:

$$
\bar{A}^{c}(t)=\frac{1}{(1+c t)^{2}}, \quad \bar{B}^{c}(t)=\frac{-2 c-c^{2} t}{(1+c t)^{4}} .
$$

It can be see also from (3.8) that the Euclidean geometry is a fixed point of the transformation $F \rightarrow \bar{F}$ when $\bar{\alpha}=(1, \ldots, 1)$. The Riemannian metric of (3.27) respectively $(3.29)$ is:

$$
g_{i j}=A \delta_{i j}+B x^{i} x^{j}, \quad \bar{g}_{i j}=\bar{A} \delta_{i j}+\bar{B} x^{i} x^{j} .
$$

\section{Finslerian flows}

For the given manifold $M$ let Finsler $(M \times \mathbb{R})$ be the infinite space of all possible time-dependent Finslerian metrics on $M$ as well as $T_{2}^{s}(T M \times \mathbb{R})$ the space 
of all time-dependent symmetric tensor fields of $(0,2)$-type on $T M$. Following the theory of geometric (more precisely Riemannian) flows we introduce:

Definition 4.1 A Finslerian flow on $M$ is a dynamical system modeled by the partial differential equations:

$$
\partial_{t} g_{t}=\mathcal{F}\left(g_{t}\right)
$$

where $\mathcal{F}:$ Finsler $(M \times \mathbb{R}) \rightarrow T_{2}^{s}(T M \times \mathbb{R})$ is a given map and $g_{t}$ is a family of Finslerian metrics depending on the parameter $t$ belonging to the interval $I \subseteq \mathbb{R}$.

Examples 4.2 i) (Special Riemannian flows) If we restrict the functional $\mathcal{F}$ to Riemann $(M \times \mathbb{R})$ to be the $(-2)$ Ricci curvature then we obtain the famous Ricci flow provided the proof of two outstanding conjectures: Poincaré Conjecture and Thurston Geometrization Conjecture. For a relationship between Randers metrics and Ricci solitons via the Zermelo navigation problem see $[6]$.

Other famous Riemannian flows are: the Calabi flow and the Yamabe flow. ii) (Poincaré flow) The discrete Poincaré flow of Example 3.2 can be considered as part of the Riemannian flow:

$$
g(t)=\frac{1}{\left(x^{m}\right)^{t}}\left(d\left(x^{1}\right)^{2}+\ldots+d\left(x^{m}\right)^{2}\right)
$$

which satisfies: $\partial_{t} g_{t}=\left(-\ln x^{m}\right) \cdot g_{t}$. Thus, we have the Poincaré flow:

$\mathcal{F}_{\text {Poincare }}: \operatorname{Riemann}\left(H_{+}^{m} \times \mathbb{R}\right) \rightarrow T_{s}^{2}\left(T H_{+}^{m} \times \mathbb{R}\right), \mathcal{F}_{\text {Poincare }}(g)=\left(-\ln x^{m}\right) \cdot g$

which is a conformal flow.

iii) (Randers flow) In the Randers example 3.4 we re-denote $b$ as $t$. Then the Randers metric (3.18) is, for $m=2$ :

$$
\begin{gathered}
g(t)_{11}=1+t^{2}+t \frac{2\left(y^{1}\right)^{3}+3 y^{1}\left(y^{2}\right)^{2}}{\left(\left(y^{1}\right)^{2}+\left(y^{2}\right)^{2}\right)^{\frac{3}{2}}}, g(t)_{12}=\frac{t\left(y^{2}\right)^{3}}{\left(\left(y^{1}\right)^{2}+\left(y^{2}\right)^{2}\right)^{\frac{3}{2}}}, \\
g(t)_{22}=1+\frac{t\left(y^{1}\right)^{3}}{\left(\left(y^{1}\right)^{2}+\left(y^{2}\right)^{2}\right)^{\frac{3}{2}}} .
\end{gathered}
$$

equivalently:

$$
g(t)=g_{t}=I_{2}+\frac{t}{\left[\left(y^{1}\right)^{2}+\left(y^{2}\right)^{2}\right]^{\frac{3}{2}}}\left(\begin{array}{cc}
2\left(y^{1}\right)^{3}+3 y^{1}\left(y^{2}\right)^{2} & \left(y^{2}\right)^{3} \\
\left(y^{2}\right)^{3} & \left(y^{1}\right)^{3}
\end{array}\right)+t^{2}\left(\begin{array}{ll}
1 & 0 \\
0 & 0
\end{array}\right) .
$$


It follows a Finslerian flow with $g(0)=I_{2}$. From $y^{2}=y^{1} \sqrt[3]{\frac{g_{12}}{g_{22}}}$ we get:

$\mathcal{F}_{\text {Randers }}(g)=\frac{1}{\left[\left(g_{12}\right)^{\frac{2}{3}}+\left(g_{22}\right)^{\frac{2}{3}}\right]^{\frac{3}{2}}}\left(\begin{array}{cc}2 g_{22}+3 \sqrt[3]{g_{12}^{2}} g_{22} & g_{12} \\ g_{12} & g_{22}\end{array}\right)+2 t\left(\begin{array}{ll}1 & 0 \\ 0 & 0\end{array}\right)$.

The associated wave system is: $\partial_{t t} g_{t}=2\left(\begin{array}{ll}1 & 0 \\ 0 & 0\end{array}\right)$. We finish this Example with the remark that time-dependent Randers metrics are recently used in the study of causal relationships on space-time manifolds in [15].

Returning to the general Finslerian framework and vector $\bar{\alpha}$ of previous Section we consider:

Definition 4.3 The Finslerian $\bar{\alpha}$-flow is that given by:

$$
\mathcal{F}(g)=\bar{g}=g^{\bar{\alpha}} .
$$

Inspired by [7] we introduce the corresponding volume variation as the smooth function $V: T M \times \mathbb{R} \rightarrow T M \times \mathbb{R}$ given by:

$$
\partial_{t} V(x, y, t)=\sum_{i, j=1}^{m} \bar{g}_{i j} g^{i j}
$$

where, as usual, $g^{i j}$ are the components of inverse $g^{-1}$.

Example 4.4 (Riemannian $\bar{\alpha}$-flow) With the notations of Example 3.2 we have:

$$
\partial_{t} a_{u v}=\sum_{i=1}^{m} \alpha^{i} a_{i u} a_{i v}
$$

with the solution:

$$
a(t)=\frac{-1}{t} \operatorname{diag}\left(\frac{1}{\alpha^{1}}, \ldots, \frac{1}{\alpha^{m}}\right)
$$

on $I=\mathbb{R}_{-}=(-\infty, 0)$. Indeed, a careful look at $(4.9)$ with respect to the powers of $t$ suggests the form:

$$
a(t)=\frac{1}{t}\left(b_{i j}(x)\right)
$$

and then we obtain:

$$
-b_{u v}=\sum_{i=1}^{m} \alpha^{i} b_{i u} b_{i v}
$$

Since $a$ is a Riemannian metric it follows that $b$ is also a metric and then it is invertible. Let us multiply (4.12) with $b^{v k}$, hence:

$$
-\delta_{u}^{k}=\sum_{i=1}^{m} \alpha^{i} b_{i u} \delta_{i}^{k}=\alpha^{k} b_{k u}
$$


with the solution: $b_{k k}=-\frac{1}{\alpha^{k}}$. We have also:

$$
\bar{a}=\frac{1}{t^{2}} \operatorname{diag}\left(\frac{1}{\alpha^{1}}, \ldots, \frac{1}{\alpha^{m}}\right), \quad a^{-1}=(-t) \cdot \operatorname{diag}\left(\alpha^{1}, \ldots, \alpha^{m}\right)
$$

and therefore:

$$
\partial_{t} V(x, t)=\sum_{i=1}^{m}\left(-\frac{1}{t}\right)=-\frac{m}{t}>0
$$

which means that the Riemannian $\bar{\alpha}$-flow is expanding with the volume variation (not depending of point):

$$
V(x, t)=V(t)=-m \ln (-t) .
$$

As in the spectral geometry we ask if the flow produces some informations about the geometry of manifold. From (4.15) it results that the $\bar{\alpha}$-flow gives the dimension of manifold by:

$$
\operatorname{dim} M=-V(-e) .
$$

The point $t_{0}=-1 \in I$ is the unique zero of $V$ and for $\bar{\alpha}=(1, \ldots, 1)$ the solution $a(-1)$ is the Euclidean metric on $M=\mathbb{R}^{m}$.

The general Finslerian $\bar{\alpha}$-flow admits the solution:

$$
g(x, y, t)=\frac{1}{t}\left(b(x, y)-\operatorname{diag}\left(\frac{1}{\alpha^{1}}, \ldots, \frac{1}{\alpha^{m}}\right)\right)
$$

where the tensor field $b=\left(b_{i j}(x, y)\right)$ satisfies the algebraic-differential system:

$$
\frac{\delta_{u v}}{\alpha^{u}}-b_{u v}=\sum_{i=1}^{m} \alpha^{i}\left[\left(b_{i u}-\frac{\delta_{i u}}{\alpha^{u}}\right)\left(b_{i v}-\frac{\delta_{i v}}{\alpha^{v}}\right)+2\left(b_{i j}-\frac{\delta_{i j}}{\alpha^{i}}\right) \frac{\partial b_{u v}}{\partial y^{i}} y^{j}\right]
$$

with $\delta_{*}$ the Kronecker delta symbol. If $F_{b}(x, y)$ is the Finsler fundamental function yielding the metric $b$ through (2.1) then the time-dependent Finsler function $F_{g}$ generating $g(t)$ is:

$$
F_{g}(x, y, t)=\sqrt{\frac{1}{t}\left\{F_{b}^{2}(x, y)-\left[\frac{1}{2 \alpha^{1}}\left(y^{1}\right)^{2}+\ldots+\frac{1}{2 \alpha^{m}}\left(y^{m}\right)^{2}\right]\right\}}
$$

Let us remark that there are two autonomous Finsler functions in the right hand side of (4.20): $F_{b}$ and the Riemannian energy yielding the diagonal constant metric $\left(\operatorname{diag}\left(\frac{1}{\alpha^{1}}, \ldots, \frac{1}{\alpha^{m}}\right)\right)$; let us remark that this last metric is exactly the $\frac{1}{\bar{\alpha}}:=\left(\frac{1}{\alpha^{1}}, \ldots, \frac{1}{\alpha^{m}}\right)$-deformation of the ambient Euclidean metric of $M$ as 
subset of $\mathbb{R}^{m}$. In the reference [16], from two Finsler functions $F_{+}, F_{-}$, it is obtained a bi-metric:

$$
F=\sqrt{F_{+} \cdot F_{-}} .
$$

The negative result of [16] concerning the physical implications of this metric as well as the considerations of our Section 1 suggests other two deformations:

$$
F_{2, \alpha, \beta}=\sqrt{\alpha F_{+}^{2}+\beta F_{-}^{2}}, \quad F_{m, \alpha, \beta}=\sqrt[m]{\alpha F_{+}^{m}+\beta F_{-}^{m}}, \quad m \in \mathbb{N}^{*}
$$

which will be studied in a future work. Let us finish with the interplay between Euclidean and Hyperbolic geometry on $H_{+}^{m}$; more precisely we consider:

$$
F_{+}:=\frac{1}{2}\left[\left(y^{1}\right)^{2}+\ldots+\left(y^{m}\right)^{2}\right], \quad F_{-, p}:=\frac{1}{2\left(x^{m}\right)^{p}}\left[\left(y^{1}\right)^{2}+\ldots+\left(y^{m}\right)^{2}\right] .
$$

Hence the bi-metric Finsler function (3.21) is exactly $F_{-, p / 2}$ and we recover the discrete (dyadic) Poincaré flow of Example 2.2.

\section{References}

[1] https://en.wikipedia.org/wiki/Affine_manifold

[2] Y. Akrami; T. S. Koivisto; A. R. Solomon, The nature of spacetime in bigravity: two metrics or none?, Gen. Relativity Gravitation, 47(2015), no. 1, Art. 1838, 6 pp. MR3284341

[3] A. Bóna; M. A. Slawinski, Wavefronts and rays as characteristics and asymptotics, Hackensack, NJ: World Scientific, 2011. Zbl 1248.35001

[4] O. Constantinescu; M. Crasmareanu, Examples of conics arising in twodimensional Finsler and Lagrange geometries, An. Stiint. Univ. "Ovidius" Constanta Ser. Mat., 17(2009), no. 2, 45-59. MR2561008 (2010j:53031)

[5] B. Chow, P. Lu, L. Ni, Hamilton's Ricci flow, Graduate Studies in Mathematics, 77, A.M.S. Providence, RI; Science Press, New York, 2006. MR2274812 (2008a:53068)

[6] M. Crasmareanu, New tools in Finsler geometry: stretch and Ricci solitons, Math. Rep. (Bucur.), 16(66)(2014), no. 1, 83-93. MR3304401

[7] M. Crasmareanu; M. Kitayama, Transformations of generalized Lagrange metrics, Tensor, 62(2000), no. 2, 167-175. Zbl 1121.53309 
[8] S.-L. Eriksson; H. Orelma, Hyperbolic Laplace operator and the Weinstein equation in $\mathbb{R}^{3}$, Adv. Appl. Clifford Algebr., 24(2014), no. 1, 109-124. MR3167591

[9] R. Miron, Sur les connexions pseudo-Euclidiennes des espaces de Finsler à métrique indéfinie, Acad. R. P. Române Fil. Iasi Stud. Cerc. Sti. Mat., 12(1961) 125-134. MR0131853 (24 \#A1700)

[10] R. Miron; M. Anastasiei, The geometry of Lagrange spaces: theory and applications, Kluwer Academic Publishers, FTPH no. 59, 1994. MR1281613 (95f:53120)

[11] R. Miron; D. Brânzei, Backgrounds of arithmetic and geometry, Series in Pure Mathematics, 23, World Scientific Publishing Co. Inc., River Edge, NJ, 1995. MR1412145 (97j:51001)

[12] R. Miron; B. T. Hassan, Gravitation and electromagnetism in FinslerLagrange spaces with $(\alpha, \beta)$-metrics, Tensor, 73(2011), no. 1, 75-86. MR2986316

[13] P. Petersen, Riemannian geometry, Second edition. Graduate Texts in Mathematics, 171, Springer, New York, 2006. MR2243772 (2007a:53001)

[14] V. Rovenski, Modeling of curves and surfaces with MATLAB, Springer Undergraduate Texts in Mathematics and Technology, Springer Berlin, 2010. Zbl 1206.65061

[15] J. Skakala; M. Visser, The causal structure of spacetime is a parameterized Randers geometry, Classical Quantum Gravity, 28(2011), no. 6, 065007, 7 pp. MR2773467 (2012d:53065)

[16] J. Skakala; M. Visser, Bi-metric pseudo-Finslerian spacetimes, J. Geom. Phys., 61(2011), no. 8, 1396-1400. MR2802480 (2012e:53143)

Mircea CRASMAREANU,

Faculty of Mathematics,

University "Al. I.Cuza",

700506 Iasi, Romania.

Email: mcrasm@uaic.ro

http://www.math.uaic.ro/ morasm 